\title{
Immune complex negatively regulates toll-like receptor 3-triggered tumour necrosis factor $\alpha$ production in B cells
}

\author{
LI QIAN ${ }^{1,2,3}$, WENYAN CHEN ${ }^{l}$, SHAOQING WANG ${ }^{l}$, YANG LIU ${ }^{1}$, XIAOQIN JIA ${ }^{l}, Y I F U^{l}$, \\ WEIJUAN GONG ${ }^{1}$, FANG TIAN ${ }^{I}$ \\ ${ }^{1}$ Laboratory of Immunology, Yangzhou University School of Medicine, Yangzhou, China \\ ${ }^{2}$ Jiangsu Co-innovation Center for Prevention and Control of Important Animal Infectious Diseases and Zoonoses, Yangzhou, China \\ ${ }^{3}$ Jiangsu Key Laboratory of Integrated Traditional Chinese and Western Medicine for Prevention and Treatment of Senile Diseases, \\ Yangzhou,China
}

\begin{abstract}
Inappropriate activation of toll-like receptor 3 (TLR3) has been implicated in the pathogenesis of autoimmune diseases, so the negative regulation of TLR3-triggered immune response has received increasing attention. Nonpathogenic immune complex (IC) has been used as treatment for many inflammatory and autoimmune diseases. However, the role of IC in the regulation of TLR3-triggered immune responses and the underlying mechanisms need to be investigated. In this study we demonstrate that IC or intravenous immunoglobulin (Ig) stimulation of B cells attenuates polyinosinic:polycytidylic acid (poly I:C)-induced CD40 expression; IC, but not Ig, can significantly inhibit poly I:C-induced pro-inflammatory tumour necrosis factor $\alpha(T N F-\alpha)$ production by B cells. Moreover, IC/Ig stimulation does not alter the expression of TLR 3 in B cells. Further experiments suggest that receptor for the Fc portion of IgGIIb $(F c \gamma R I I b)$ is involved in the suppressive effect of IC on TLR3-mediated TNF- $\alpha$ production, but not CD40 expression. Thus, we provide a new means of negative regulation of TLR3-triggered immune responses in B cells via FcyRIIb, and we provide a new mechanistic explanation of the therapeutic effect of nonpathogenic IC on inflammatory or autoimmune diseases.
\end{abstract}

Key words: B cells, FcyRIIb, immune complex, Toll-like receptor.

(Centr Eur Immunol 2017; 42 (3): 223-230)

\section{Introduction}

Toll-like receptors (TLRs) are pivotal components of the innate immune response, which is responsible for discriminating invading microbial pathogens $[1,2]$. Toll-like receptors also initiate adaptive immune responses and lead to amplified immune response against microbial pathogens by activating $\mathrm{T}$ and $\mathrm{B}$ cells [3-5]. For example, most TLR ligands, including viral double-stranded RNA (dsRNA), activate $\mathrm{B}$ cells. This is characterised by the up-regulation of MHC class II, induction of co-stimulatory molecules, and secretion of pro-inflammatory cytokines, which can presumably increase their potential activity for antigen presentation $[6,7]$.

But excessive responses to TLR agonists may induce immunopathological conditions, such as autoimmune diseases [8-11]. For example, expression of TLR3 was significantly higher in rheumatoid arthritis (RA) synovial fibroblasts compared with healthy controls or patients with non-inflammatory arthritis. Toll-like receptor 3 activation increased disease severity in the rat pristane-induced arthritis and rat collagen-induced arthritis (CIA) models, and small interfering RNA targeting TLR3 in vivo reduced disease severity [12]. Treatment of pristane-induced arthritis in rats with a microRNA-26a decreased TLR3 expression and disease symptoms, confirming a role for TLR3 in this model [13]. Therefore, inhibition of TLR3 activation may have therapeutic benefit in such autoimmune diseases.

Receptors for the Fc portion of IgG (Fc $\gamma \mathrm{Rs})$ are important in both promoting and regulating the immune and inflammatory response to immune complex (IC) [14, 15]. Most Fc $\gamma$ Rs are activating receptors and Fc $\gamma$ RIIb is the only Fc $\gamma \mathrm{R}$ that has an inhibitory function. Fc $\gamma \mathrm{RIIb}$ is the only Fc $\gamma \mathrm{R}$ expressed by B cells, and engagement of Fc $\gamma-$ RIIb can negatively regulate $B$ cell activation $[16,17]$. In accordance with its inhibitory function, Fc $\gamma$ RIIb deficiency increases disease severity in CIA and Fc $\gamma$ RIIb B cell transgenic mice show a reduction in disease severity and in collagen-specific $\operatorname{IgG}$ titres [18]. Interaction between

Correspondence: Li Qian, Laboratory of Immunology, Yangzhou University School of Medicine, 11 Huaihai Road, 225001 Yangzhou,

China, e-mail: qianl@yzu.edu.cn

Submitted: 4.05.2016; Accepted: 12.09.2016 
nonpathogenic soluble IC and Fc $\gamma$ RIIb has been demonstrated to be remarkably effective in the treatment of many autoimmune and inflammatory diseases [19-22]. However, the role of soluble IC and Fc $\gamma$ RIIb in the regulation of TLR3-mediated activation of B cells and their influence on the expression of TLR3 remain to be further identified.

In this study, we demonstrated that soluble IC (composed of OVA Ag and anti-OVA mAb) can negatively regulate TLR3-triggered activation in B cells. Most notably, our experiments demonstrate that Fc $\gamma$ RIIb is responsible for the suppressive effect of IC on TLR3-mediated TNF- $\alpha$ production in B cells. Thus, we provide a new means of negative regulation of TLR3-triggered immune responses in B cells via Fc $\gamma$ RIIb.

\section{Material and methods}

\section{Mice}

C57BL/6J mice and FcyIIb-deficient mice were obtained from the Experimental Animal Centre of Yangzhou University (Jiangsu, China) and Jackson Laboratory (Bar Harbor, ME, USA), respectively, and used at the age of 5-6 weeks. Animal experiments were performed in accordance with the National Institutes of Health Guide for the Care and Use of Laboratory Animals, with the approval of the Animal Welfare Association of Jiangsu province (Jiangsu, China). The study was approved by the Ethics Committee of Yangzhou University.

\section{Preparation of immune complex}

Soluble IC was prepared as previously described [22]. Briefly, OVA stock (Sigma-Aldrich, St. Louis, MO, USA) was prepared to $10 \mathrm{mg} / \mathrm{ml}$ using PBS, and then $50 \mu \mathrm{g}$ of OVA was incubated with $500 \mu \mathrm{g}$ mouse-derived anti-OVA monoclonal antibody (mAb; IgG1, Sigma-Aldrich) at $37^{\circ} \mathrm{C}$ for $1 \mathrm{~h}$ to obtain anti-OVA IC. IC (OVA/anti-OVA mAb) was used at a final concentration of $10 \mu \mathrm{g} / \mathrm{ml} \mathrm{OVA} \mathrm{plus}$ $100 \mu \mathrm{g} / \mathrm{ml}$ anti-OVA mAb. Endotoxin contamination in OVA and anti-OVA reagent was measured using Limulus amebocyte lysate (ZhanJiang A\&C Biological, Shanghai, China), and the level of endotoxin in $10 \mu \mathrm{g}$ of OVA plus $100 \mu \mathrm{g}$ of anti-OVA mAb was lower than $0.03 \mathrm{EU}$.

\section{Isolation of mouse $\mathrm{CD19}^{+} \mathrm{B}$ cells}

Spleen-derived B cells or Fc $\gamma \mathrm{IIb}^{-/-} \mathrm{B}$ cells were enriched using CD19-conjugated microbeads as recommended by the manufacturer (Miltenyi Biotec, Bergisch Gladbach, Germany). The purity of the isolated mouse CD19 cells was $>96 \%$, as analysed by flow cytometry. The isolated B cells were cultured in RPMI1640 medium supplemented with $10 \%$ FBS, 2 mM L-glutamine (Sigma-Aldrich), $100 \mathrm{U} / \mathrm{ml}$ penicillin, and $100 \mu \mathrm{g} / \mathrm{ml}$ streptomycin (Gibco RBL, Grand Island, NY, USA).

\section{$\mathrm{CD19}^{+} \mathrm{B}$ cells stimulation}

Purified splenic B cells or Fc $\gamma \mathrm{IIb}^{-/}$B cells were plated in 96-well flat-bottom plates at a density of $2.0 \times 10^{5}$ per well, and then stimulated with IC, Ig (anti-OVA mAb, 100 $\mu \mathrm{g} / \mathrm{ml})$, poly I:C (10 $\mu \mathrm{g} / \mathrm{ml})$, poly I:C plus Ig, or poly I:C plus IC. Poly I:C were purchased from InvivoGen (San Diego, CA, USA).

\section{Measurement of interleukin 6 and tumour necrosis factor $\alpha$}

At $24 \mathrm{~h}, \mathrm{~B}$ cell culture supernatants were collected and measured for IL- 6 and TNF- $\alpha$ by ELISA kit (R\&D Systems, Minneapolis, MN) according to the manufacturer's instructions.

\section{Flow cytometric analysis}

At $24 \mathrm{~h}, \mathrm{~B}$ cells were washed with ice-cold PBS buffer and then stained with antibodies to CD19-APC, CD40-PE, CD86-PE, MHC II (Ia $\left.{ }^{\mathrm{b}}\right)$-PE (BD Biosciences), or TLR3PE (BioLegend, San Diego, CA, USA) for $30 \mathrm{~min}$ at $4^{\circ} \mathrm{C}$. For intracellular TLR3 staining, cells were stained with antibody to CD19-APC and then fixed and permeabilised with a Cytofix/Cytoperm kit according to BD Biosciences. Permeabilised cells were incubated with PE-conjugated anti-TLR3 mAb. Data were collected using FACS Calibur (BD Biosciences) and analysed by FlowJo software.

\section{Statistics}

Data are presented as mean \pm SD. Statistical comparisons were made using GraphPad PRISM software. A twotailed Student $t$ test was applied. $P<0.05$ was considered to indicate statistically significant differences.

\section{Results}

\section{Immune complex/Ig stimulation inhibits TLR3-mediated CD40 expression on B cells}

First, we observed the effect of IC on poly I:C (TLR3 agonist)-induced membrane molecule expression of $\mathrm{B}$ cells. We found that B cells did not display an altered expression of CD86 and MHC II upon stimulation with IC or without IC in the presence of poly I:C (Fig. 1A, B). Interestingly, the combination of IC and poly I:C resulted in a decrease in the expression of CD40 by B cells, as compared with that observed in B cells stimulated with poly I:C alone (Fig. 1C). Furthermore, we observed the effect of free intravenous immunoglobulin (Ig, anti-OVA mAb) on poly I:C-induced CD40 expression by B cells. We found that Ig could also reduce poly I:C-induced CD40 expression (Fig. 1D). Taken together, these results suggested that IC/Ig stimulation of B cells can inhibit poly I:C-induced CD40 expression. 

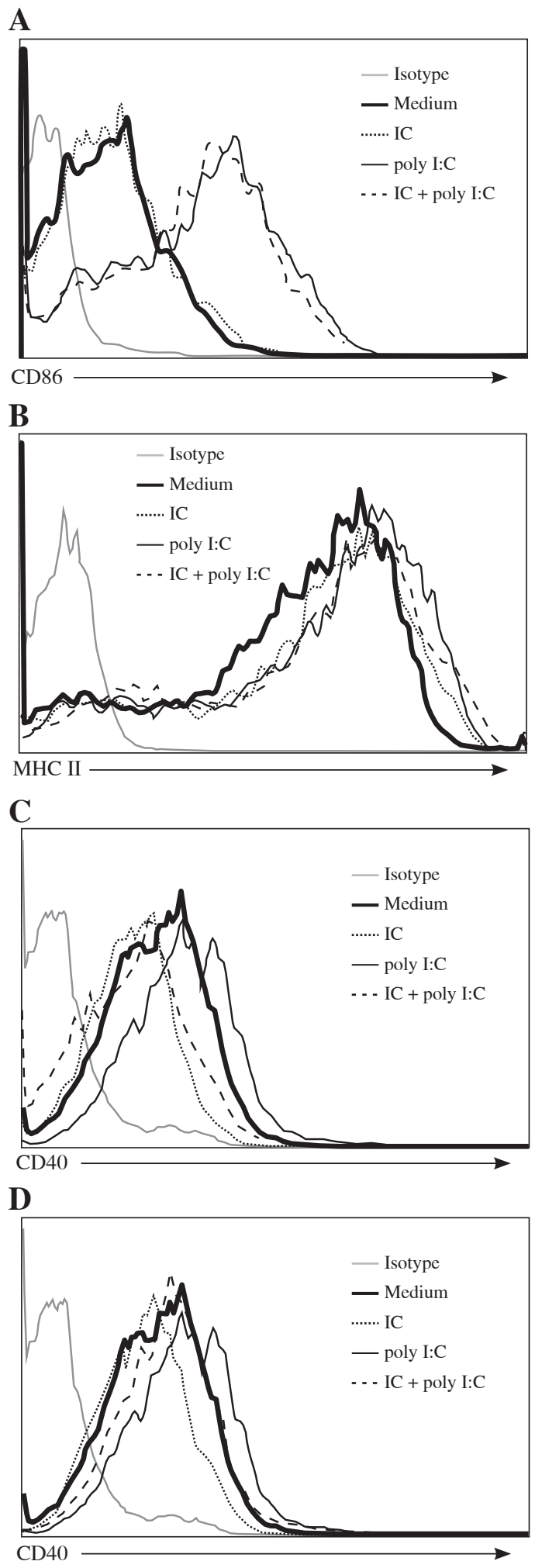
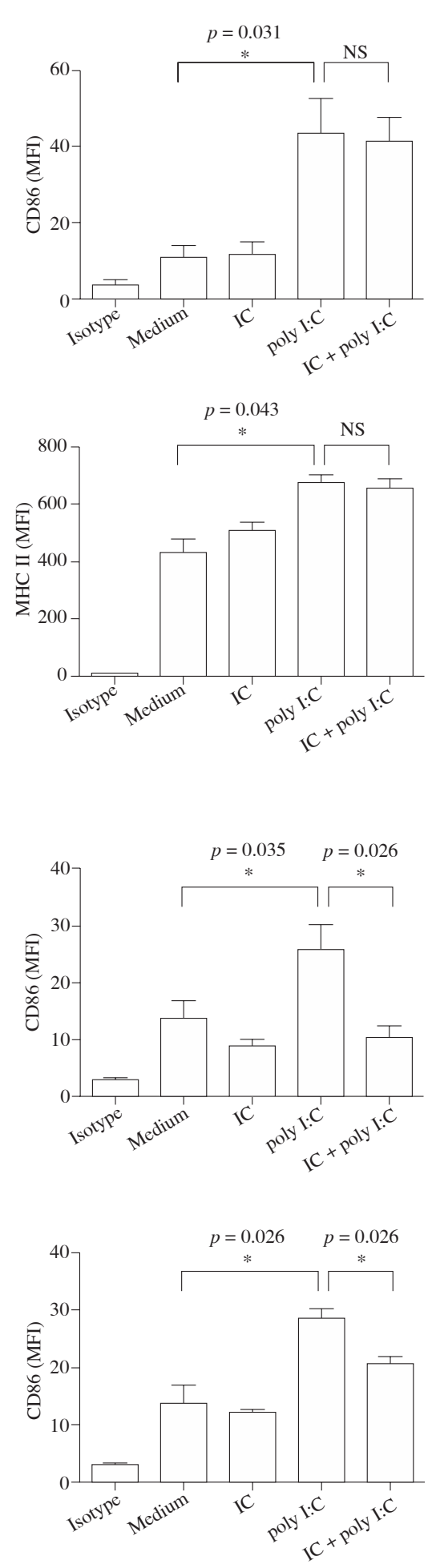

NS - not significant

Fig. 1. IC/Ig inhibits poly I:C-induced CD40 expression of splenic B cells. Freshly purified splenic B cells were stimulated for $24 \mathrm{~h}$ with IC, poly I:C, or poly I:C plus IC. Expression of CD86 (A), MHC II (B), and CD40 (C) on CD19+ B cells was detected by flow cytometry. D) Freshly purified splenic B cells were stimulated for $24 \mathrm{~h}$ with Ig, poly I:C, or poly I:C plus Ig. Expression of CD40 on CD19+ B cells was detected by flow cytometry. Left panels, histograms represent one of three independent experiments. Right panels, numbers indicate mean fluorescence intensity (MFI) of test samples. Data are presented as the means $\pm \mathrm{SD}, * p<0.05$ 


\section{Immune complex stimulation inhibits TLR3- mediated TNF- $\alpha$ secretion of $B$ cells}

To further determine whether the poly I:C-induced production of pro-inflammatory cytokines in $\mathrm{B}$ cells is affected by IC, we stimulated B cells with a combination of poly I:C and IC and compared them with B cells stimulated with poly I:C alone. We found that both unstimulated and stimulated $\mathrm{B}$ cells failed to release any detectable IL-12p70, IP-10, IFN-g, or IL-17A (data not shown). Poly I:C alone could significantly induce the production of IL-6 and TNF- $\alpha$ from B cells (Fig. 2A, B). There was no significant difference in production of IL- 6 by $\mathrm{B}$ cell stimulation with or without IC in the presence of poly I:C (Fig. 2A), suggesting that IC stimulation of B cells cannot inhibit poly I:C-induced IL-6 production. However, IC clearly inhibited the production of TNF- $\alpha$ from poly I:C-activated B cells (Fig. 2B). Moreover, we found that Ig could not reduce poly I:C-induced TNF- $\alpha$ production (Fig. 2C). Taken together, these results suggested that treatment of B cells with IC can significantly suppress poly I:C-induced TNF- $\alpha$ production.

\section{Immune complex/Ig stimulation has no effect on TLR3 expression in B cells}

Then we wondered whether IC/Ig negatively regulates TLR3-mediated immune response by down-regulation of TLR3 expression in B cells. We stimulated B cells with IC or Ig and then detected the expression of TLR3 in B cells.
As shown in Figure 3, both cell surface and intracellular TLR3 expression in B cells remained unchanged after IC or Ig stimulation. These data suggest that IC/Ig-mediated suppression of TLR3-mediated immune response in B cells is not due to down-regulation of TLR3 expression.

\section{Fc $\gamma \mathbf{R I I b}$ is responsible for the suppressive effect of immune complex on poly I:C-triggered TNF- $\alpha$ production}

It has been well-established that Fc $\gamma I I b$, the unique inhibitory Fc $\gamma \mathrm{R}$ expressed on B cells, plays a critical role in negative regulation of immune responses, and IC can bind to Fc $\gamma$ RIIb to exert its biological activities [17, 22]. We then wondered whether Fc $\gamma$ RIIb was responsible for IC/Ig-mediated suppression of TLR3 signalling in B cells. So, we analysed the CD40 expression and TNF- $\alpha$ secretion by poly I:C-stimulated $\mathrm{Fc} \gamma \mathrm{RIIb}^{-/} \mathrm{B}$ cells treated with or without IC or Ig. As shown in Fig. 4A, B, IC/Ig stimulation of $\mathrm{Fc} \gamma \mathrm{RIIb}^{-/} \mathrm{B}$ cells significantly suppressed poly I:C-induced CD40 expression, similar to that of wild type B cells (Fig. 1C, D). These results suggest that Fc $\gamma$ RIIb does not play an important role in the IC/Ig-mediated inhibition of CD40 expression. Interestingly, IC treatment lost its inhibitory effect poly I:C-induced TNF- $\alpha$ secretion when Fc $\gamma \mathrm{RIIb}^{--} \mathrm{B}$ cells were used (Fig. 4C), indicating that Fc $\gamma$ RIIb is responsible for the suppressive effect of IC on TLR3-mediated TNF- $\alpha$ secretion.
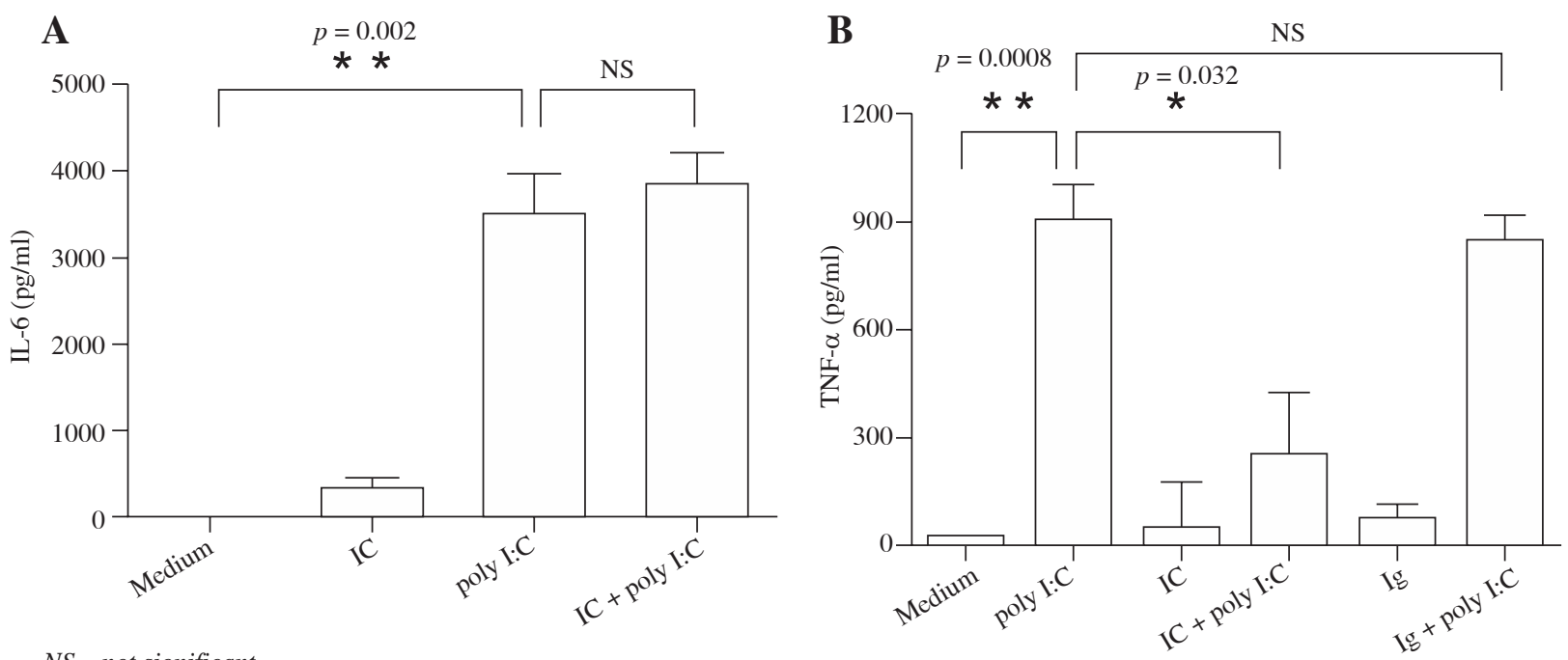

NS - not significant

Fig. 2. IC inhibits poly I:C-induced TNF- $\alpha$ secretion from splenic B cells. A) Freshly purified splenic B cells were stimulated for $24 \mathrm{~h}$ with IC, poly I:C, or poly I:C plus IC. IL-6 in the supernatants was measured by ELISA. B) Freshly purified splenic B cells were stimulated for $24 \mathrm{~h}$ with IC/Ig, poly I:C, or poly I:C plus IC/Ig. Culture supernatants were collected and assayed for TNF- $\alpha$ production by ELISA. Results are representative of three independent experiments. Data are expressed as mean $\pm \mathrm{SD}, * p<0.05 ; * * p<0.01$ 
A

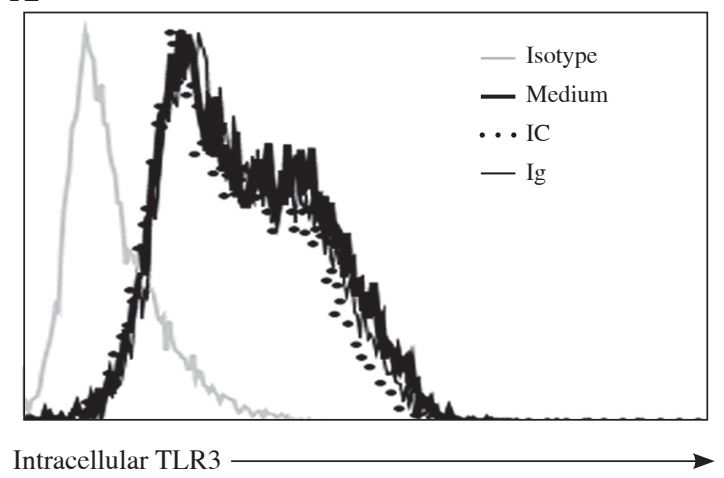

\section{B}

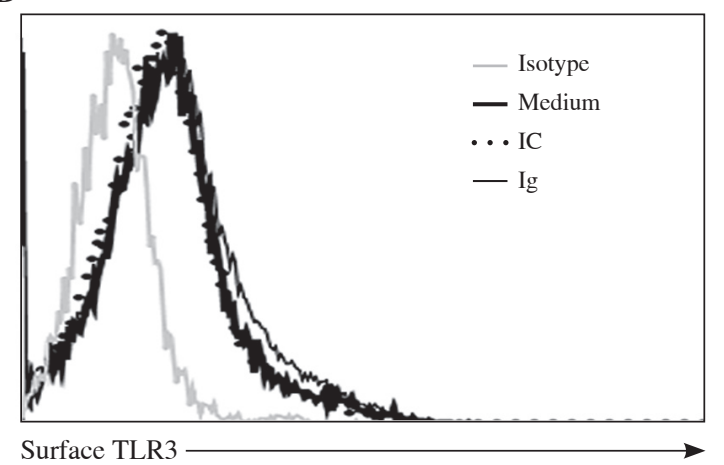

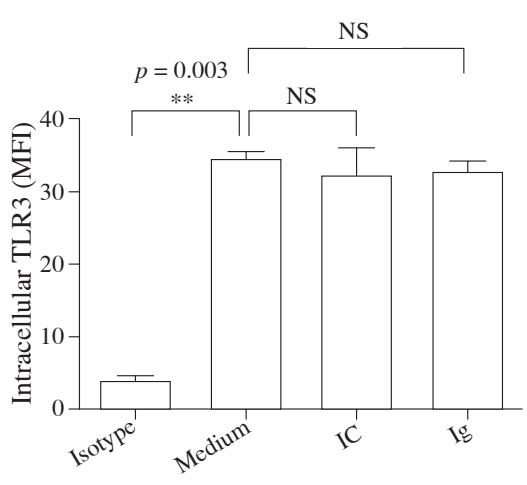

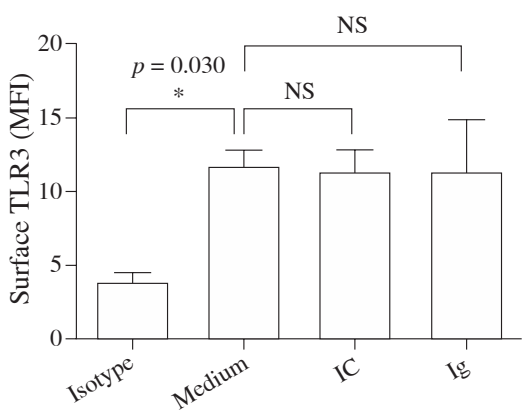

NS - not significant

Fig. 3. IC/Ig has no effect on TLR3 expression in splenic B cells. Freshly purified splenic B cells were stimulated for $24 \mathrm{~h}$ with IC or Ig. A) TLR3 in CD19+ B cells was detected by flow cytometry with intracellular staining. B) Expression of TLR3 on CD19+ B cells was detected by flow cytometry. Left panels, histograms represent one of three independent experiments. Right panels, numbers indicate MFI of test samples. Data are presented as the means $\pm \mathrm{SD}, * p<0.05$; $* * p<0.01$

\section{Discussion}

Toll-like receptor 3 has been identified as an endosomal or cell-surface receptor that recognises and mediates responses to viral dsRNA or poly I:C, an analogue of viral dsRNA [23]. It has been demonstrated that B cells express TLR3, and that they can be activated via TLR3 ligands [24]. This was confirmed by our finding that poly I:C induced up-regulation of MHC II, CD86, and CD40, and production of TNF- $\alpha$ and IL- 6 from B cells.

Intravenous Ig has been used in an increasing number of immune-mediated conditions, including acute and chronic/relapsing autoimmune diseases and systemic inflammatory disorders $[25,26]$. Recent data indicate that IgG reactive with soluble antigens (soluble IC) can mimic the therapeutic effects of IV Ig in treating some autoimmune diseases [19]. It was previously reported that IV Ig-induced down-regulation of MHC II and CD86 on dendritic cells resulted in the inhibition of autoreactive and alloreactive T-cell activation and proliferation [27]. However, our results showed that the expression of MHC II and CD86 was not modified in poly I:C-activated B cells in the presence of IC. These differences could be due to differences related to the cell types involved. Interestingly, we showed that IC/Ig inhibited CD40 upregulation on poly I:C-activated B cells. Evidence from several sources supports the key role of CD40L/CD40 interactions in initiating and/or propagating chronic inflammatory autoimmune diseases such as rheumatoid arthritis (RA) [28]. The administration of antagonistic anti-CD40L mAbs significantly ameliorates collagen-induced arthritis [29], indicating that disruption of this pathway could be a beneficial treatment in RA. Furthermore, highly elevated levels of tumour necrosis factor $\alpha$ (TNF- $\alpha$ ) and TNF- $\alpha$ polymorphisms have been associated with various disease states, such as RA [10, 30]. Biological agents targeting TNF- $\alpha$ show significant clinical benefit and halt radiographic progression, confirming the key role of TNF- $\alpha$ in RA [30, 31]. 
A

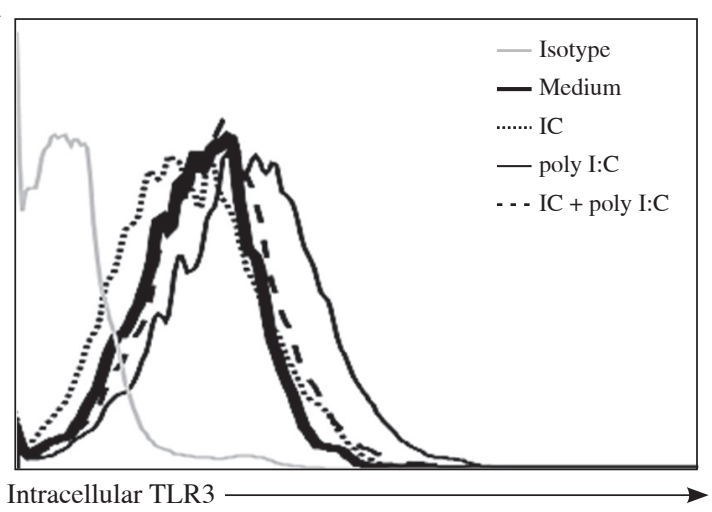

B

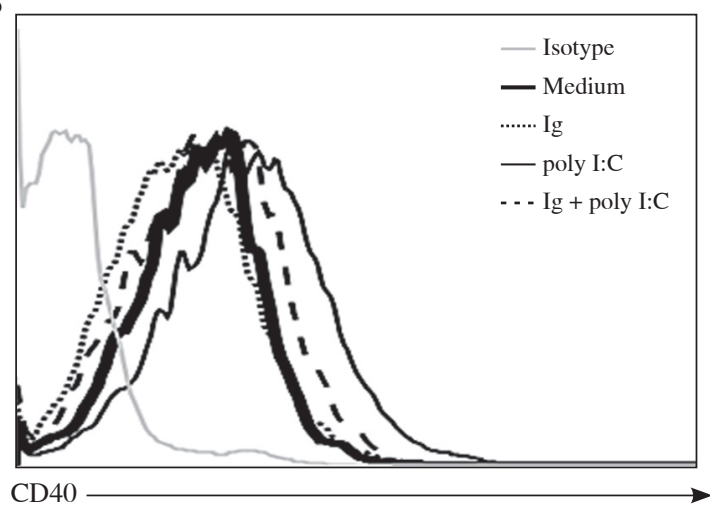

$\mathrm{Fc}_{\mathrm{RIIb}}{ }^{--}$

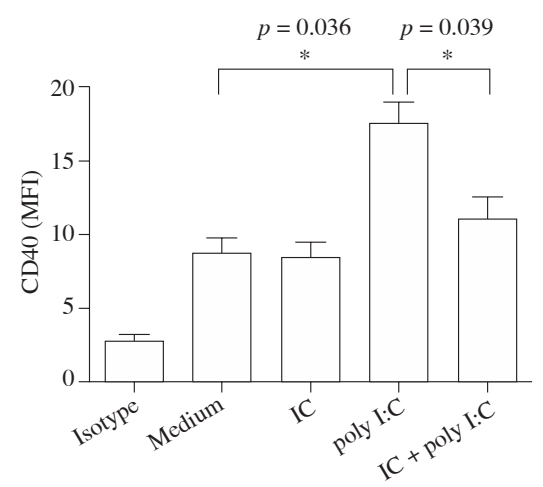

$\mathrm{Fc} \gamma \mathrm{RII}^{--}$

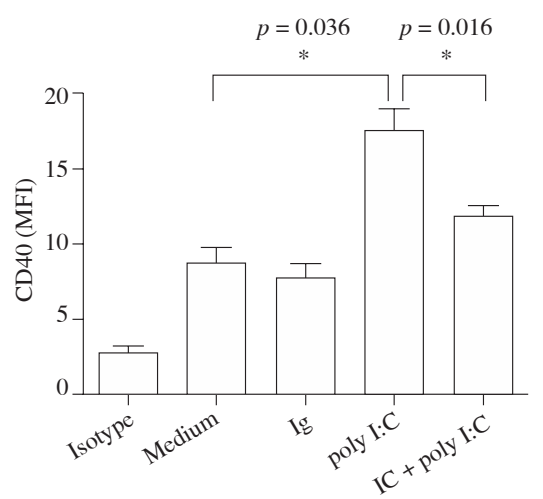

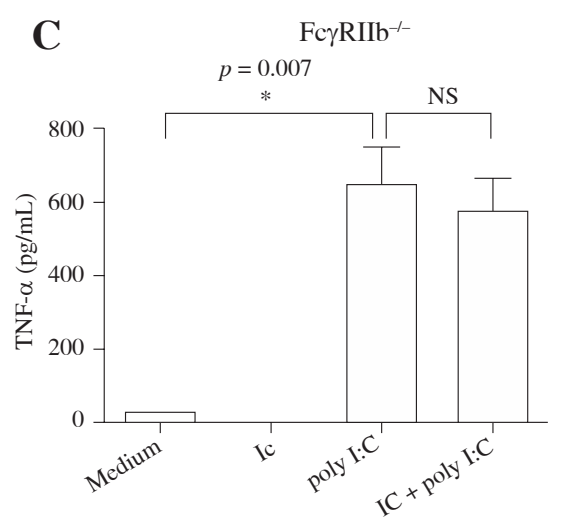

NS - not significant

Fig. 4. IC inhibits TNF- $\alpha$ production from poly I:C-stimulated B cells via inhibitory Fc $\gamma I I b$. A, B) Freshly purified splenic $\mathrm{Fc}_{\mathrm{III}}{ }^{-1} \mathrm{~B}$ cells were stimulated with IC/Ig, poly I:C, or poly I:C plus IC/Ig. After $24 \mathrm{~h}$, cells were collected and analysed by flow cytometry for CD40 expression. Left panels, histograms represent one of three independent experiments. Right panels, numbers indicate MFI of test samples. Data are presented as the means $\pm \mathrm{SD},{ }^{*} p<0.05$. C) Freshly purified splenic $\mathrm{Fc}_{\mathrm{III}} \mathrm{Il}^{--} \mathrm{B}$ cells were stimulated with IC, poly I:C, or poly I:C plus IC. Culture supernatants were collected at $24 \mathrm{~h}$ and assayed for TNF- $\alpha$ by ELISA. Data are presented as the means \pm SD. $* * p<0.01$

In the present study, we demonstrate that IC decreases the expression of TNF- $\alpha$ and CD40 from poly I:C-activated B cells. In consideration of the above findings, the present study may provide an additional mechanistic explanation of the therapeutic effect of IC on autoimmune diseases such as RA.

In addition, we found that IC inhibited TLR3-mediated TNF- $\alpha$ production but did not inhibit another pro-in- 
flammatory cytokine, IL-6. We speculated that different pro-inflammatory cytokines secretion, such as IL-6 and TNF- $\alpha$, may be regulated by different signalling pathways in B cells. It is possible that IC might inhibit the signalling pathways responsible for TNF- $\alpha$ production but not the signalling pathways responsible for IL-6 production.

The precise mechanism by which IC/Ig exerts its action on B cells is not yet clear. We tried to investigate whether IC/Ig inhibits poly I:C-induced immune response by down-regulation of TLR3 expression in B cells. However, we did not observe the altered expression of TLR3 in B cells or poly I:C-activated B cells by IC/Ig (data not shown). Next, we investigated the role of Fc $\gamma$ RIIb in IC-mediated inhibition of B cell activation. As expected, we found that ICs lost their inhibitory effect on poly I:C -induced expression of TNF- $\alpha$ when $\mathrm{Fc} \gamma \mathrm{RIIb}^{-/-} \mathrm{B}$ cells were used, suggesting that IC negatively regulates poly I:C-induced TNF- $\alpha$ secretion from B cells through its interaction with Fc $\gamma$ RIIb. However, we found that Fc $\gamma$ RIIb was not responsible for the inhibitory effect of IC/Ig on CD40 expression. A recent study demonstrated that IV Ig can exert its action on B cells through binding of its sialic-acid-bearing glycans to the negative regulator CD22 [32]. So, we speculate that other receptor(s) such as CD22 might be involved in IC-induced inhibition of TLR3 signalling, which may also account for the reason why IC and Ig exert different regulatory roles in poly I:C-activated B cells. However, which receptor(s) is (are) involved needs to be further investigated.

In summary, in this study we have provided novel insights for the mechanisms underlying the negative regulation of TLR3-triggered immune responses in B cells by IC. It also contributes to a better understand of the mechanisms of clinical administration of IV Ig for prevention of serious inflammation or treatment of autoimmune diseases.

We thank Maozhi Hu for excellent technical assistance.

This study was supported by the National Natural Science Foundation of China (81001308, 81373130, 81273214, 81101265, 81172785), the Natural Science Foundation of Jiangsu Province (BK2010315), the Science and Technology Innovation Fund for Undergraduates of the Educational Committee of Jiangsu Province (2013), and the New Century Talents Project of Yangzhou University (2012).

The authors declare that they have no conflicting interests.

\section{References}

1. O’Neill LA, Golenbock D, Bowie AG (2013): The history of Toll-like receptors-redefining innate immunity. Nat Rev Immunol 13: 453-460.

2. Zheng Y, An H, Yao M, Hou J, et al. (2010): Scaffolding adaptor protein Gab1 is required for TLR3/4- and RIG-I-me- diated production of proinflammatory cytokines and type I IFN in macrophages. J Immunol 184: 6447-6456.

3. Reynolds JM, Dong C (2013): Toll-like receptor regulation of effector T lymphocyte function. Trends Immunol 34: 511519.

4. Mills KH (2011): TLR-dependent T cell activation in autoimmunity. Nat Rev Immunol 11: 807-822.

5. Hua Z, Hou B (2013): TLR signaling in B-cell development and activation. Cell Mol Immunol 10: 103-106.

6. Browne EP (2012): Regulation of B-cell responses by Tolllike receptors. Immunology 136: 370-379.

7. Green NM, Marshak-Rothstein A (2011): Toll-like receptor driven B cell activation in the induction of systemic autoimmunity. Semin Immunol 23: 106-112.

8. Celhar T, Magalhăes R, Fairhurst AM (2012): TLR7 and TLR9 in SLE: when sensing self goes wrong. Immunol Res 53: 58-77.

9. Mullen LM, Chamberlain G, Sacre S (2015): Pattern recognition receptors as potential therapeutic targets in inflammatory rheumatic disease. Arthritis Res Ther 17: 122.

10. Goh FG, Midwood KS (2012): Intrinsic danger: activation of Toll-like receptors in rheumatoid arthritis. Rheumatology (Oxford) 51: 7-23.

11. Zheng Y, Yang Y, Li Y, et al. (2013): Ephedrine hydrochloride inhibits PGN-induced inflammatory responses by promoting IL-10 production and decreasing proinflammatory cytokine secretion via PI3K/Akt/GSK3 $\beta$ pathway. Cell Mol Immunol 10: 330-337.

12. Thwaites R, Chamberlain G, Sacre S (2014): Emerging role of endosomal Toll-like receptors in rheumatoid arthritis. Front Immunol 5: 1.

13. Jiang C, Zhu W, Xu J, et al. (2014): MicroRNA-26a negatively regulates toll-like receptor 3 expression of rat macrophages and ameliorates pristane induced arthritis in rats. Arthritis Res Ther 16: R9.

14. Guilliams M, Bruhns P, Saeys Y, et al. (2014): The function of Fc $\gamma$ receptors in dendritic cells and macrophages. Nat Rev Immunol 14: 94-108.

15. Ravetch JV, Bolland S (2001): IgG Fc receptors. Annu Rev Immunol 19: 275-290.

16. Smith KG, Clatworthy MR (2010): FcrRIIB in autoimmunity and infection: evolutionary and therapeutic implications. Nat Rev Immunol 10: 328-343.

17. Espéli M, Smith KG, Clatworthy MR (2016): Fc $\gamma$ RIIB and autoimmunity. Immunol Rev 269: 194-211.

18. Yuasa T, Kubo S, Yoshino T, et al. (1999): Deletion of fcgamma receptor IIB renders $\mathrm{H}-2$ (b) mice susceptible to collagen-induced arthritis. J Exp Med 189: 187-194.

19. Siragam V, Brinc D, Crow AR, et al. (2005): Can antibodies with specificity for soluble antigens mimic the therapeutic effects of intravenous IgG in the treatment of autoimmune disease? J Clin Invest 115: 155-160.

20. Clynes R (2005): Immune complexes as therapy for autoimmunity. J Clin Invest 115: 25-27.

21. Van Egmond M, Vidarsson G, Bakema JE (2015): Cross-talk between pathogen recognizing Toll-like receptors and immunoglobulin Fc receptors in immunity. Immunol Rev 268: 311-327.

22. Zhang Y, Liu S, Liu J, et al. (2009): Immune Complex/Ig negatively regulate TLR4-triggered inflammatory response in macrophages through FcrRIIb-dependent PGE2 production. J Immunol 182: 554-562. 
23. Perales-Linares R, Navas-Martin S (2013): Toll-like receptor 3 in viral pathogenesis: friend or foe? Immunology 140: 153-167.

24. Marshall-Clarke S, Downes JE, Haga IR, et al. (2007): Polyinosinic acid is a ligand for toll-like receptor 3. J Biol Chem 282: $24759-24766$.

25. Schwab I., Nimmerjahn F (2013): Intravenous immunoglobulin therapy: how does IgG modulate the immune system? Nat Rev Immunol 13: 176-189.

26. Kessel A, Peri R, Haj T, et al. (2011): IVIg attenuates TLR-9 activation in B cells from SLE patients. J Clin Immunol 31: 30-38.

27. Bayry J, Lacroix-Desmazes S, Carbonneil C, et al. (2003): Inhibition of maturation and function of dendritic cells by intravenous immunoglobulin. Blood 101: 758-765.

28. Alaaeddine N, Hassan GS, Yacoub D, Mourad W (2012): CD154: an immunoinflammatory mediator in systemic lupus erythematosus and rheumatoid arthritis. Clin Dev Immunol 2012: 490148

29. Li L, Wang H, Wang B (2008): Anergic cells generated by blocking CD28 and CD40 costimulatory pathways in vitro ameliorate collagen induced arthritis. Cell Immunol 254: 39-45.

30. Mohammad MK, Morran M, Slotterbeck B, et al. (2006): Dysregulated Toll-like receptor expression and signaling in bone marrow-derived macrophages at the onset of diabetes in the non-obese diabetic mouse. Int Immunol 18: 1101-1113.

31. Iannone F, Lopalco G, Rigante D, et al. (2016): Impact of obesity on the clinical outcome of rheumatologic patients in biotherapy. Autoimmun Rev pii: S1568-9972: 30006-30004.

32. Sédté JF, Cornec D, Renaudineau Y, et al. (2010): IVIg modulates BCR signaling through CD22 and promotes apoptosis in mature human B lymphocytes. Blood 116: 1698-1704. 